
About IJMA [last updated July, $\left.1^{\text {st }}, 2021\right]$

$\checkmark$ International Journal of Medical Arts is the Official Journal of the Damietta Faculty of Medicine, AlAzhar University, Egypt

$\checkmark$ It is an International, Open Access, Double-blind, Peer-reviewed Journal

$\checkmark$ Published four times a year

$\checkmark$ The First Issue was published in July 2019

$\checkmark$ Published under the following license: Creative Commons Attribution-ShareAlike 4.0 International Public License (CC BY-SA 4.0). It had updated from the Creative Commons license [CC BY] in volume 2, Issue 4, October 2020 About IJMA

$\checkmark$ The Egyptian Knowledge Bank hosts the web site of IJMA

$\checkmark$ The Egyptian Knowledge Bank supports IJMA

$\checkmark$ IJMA follows the regulations of the International Committee of Medical Journal Editors

$\checkmark$ IJMA is indexed in the "Directory of Open Access Journals" [15 January 2021].

$\checkmark$ IJMA is indexed in JGate [29-6-2021]

$\checkmark$ IJMA is a member of the International Society of Managing and Technical Editors

$\checkmark$ Listed in "Index Copernicus", "Publons", "Academic resource index [ResearchBib]", "Electronics journal library", "Eurasian Scientific Journal Index", and "Citefactor"

$\checkmark$ IJMA introduced to the search engine [BASE] through DOAJ
Click image to reach the page

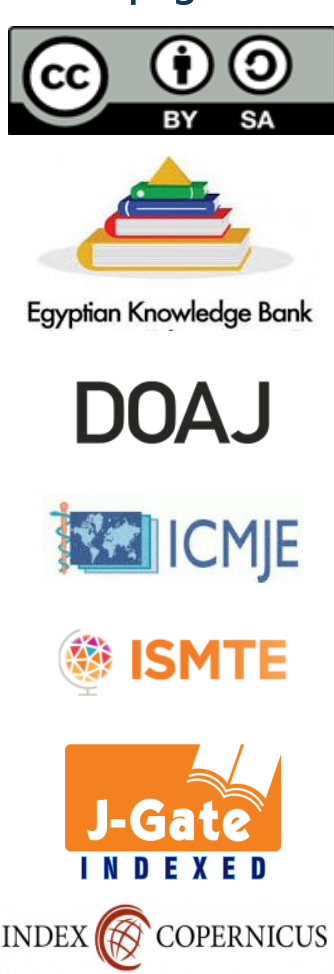

publons

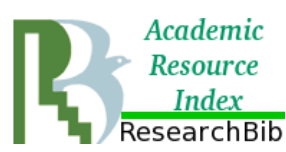

\section{EZ3 \\ .}

ESJII

CiteFactor

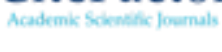

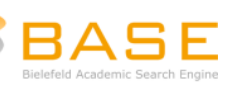




Available online at Journal Website
https://ijma.journals.ekb.eg/
Main subject [Dermatology]

Original Article

\title{
Comparative Study between Topical Latanoprost versus Topical 5- Fluorouracil after Dermabrasion for Treatment of Non-Segmental Stable Vitiligo
}

\author{
Rehab Basuny Abd El Razek [1], Zakaria Obaid [2], Osama Magdy Elshahat [2] \\ 1 Medical Administration Branch [For Girls], Al-Azhar University, Egypt. \\ 2 Department of Dermatology and Venereology, Damietta Faculty of Medicine, Al-Azhar University, Egypt
}

Corresponding author: Rehab Basuny Abd El Razek

Email: m.rehab1188@gmail.com

Submission date: March 31, 2021; Revision date: May 07, 2021; Acceptance date: May 09, 2021

\begin{abstract}
Background: Latanoprost $[L T]$, a prostaglandin F2-alpha [PGF2a] analogue used in the treatment of glaucoma. It induces skin pigmentation in guinea pigs. The rationale of trying 5-fluorouracil [5-FU] in vitiligo treatment may come from the first report of hyperpigmentation induced by 5 -FU as a side effect in the skin tumors and psoriasis treatment

The Aim of the work: Comparison between the efficacy of topical latanoprost versus topical 5 -fluorouracil after skin dermabrasion in induction of skin repigmentation in stable non-segmental vitiligo.

Patients and Methods: This study included thirty Egyptian patients, 18-60 years old, with stable non-segmental vitiligo. They were divided into: group $[A]$ patients subjected to 5 fluorouracil after dermabrasion and group $[B]$ patients subjected to latanoprost after dermabrasion then followed up every two weeks for three months.

Result: There was no statistically significant difference between both groups regarding total response to treatment and degree of repigmentation. However, there was higher response to treatment in group A than group B. In both groups patients were satisfied with the degree of improvement. As regard complication there were minimal complications only one case of koebnerization and two cases of post inflammatory hyperpigmentation

Conclusion: 5 - fluorouracil 5\% was associated with better improvement than latanoprost. However, the difference did not reach statistical significance. Patients reported high satisfaction with the two treatment modalities.
\end{abstract}

Keywords: Latanoprost; Five-fluorouracil; Vitiligo; Dermabrasion.

This is an open-access article registered under the Creative Commons, ShareAlike 4.0 International license [CC BY-SA 4.0] [https://creativecommons.org/licenses/by-sa/4.0/legalcode.

Citation: Abd El Razek RB, Obaid Z, Elshahat OM. Comparative study between Topical Latanoprost versus Topical 5 Fluorouracil after Dermabrasion for Treatment of Non-Segmental Stable Vitiligo. IJMA 2021; 3 [3] July-September: 1550-1555. [DOI: 10.21608/IJMA.2021.70410.1290].

${ }^{*}$ Main subject and any subcategories have been classified according to the research topic. 


\section{INTRODUCTION}

Vitiligo is an acquired hypomelanotic disorder. It is characterized by circumscribed depigmented macules in the skin, resulting from the destruction of functioning melanocytes and lost melanin from cutaneous epidermis [1].

Latanoprost, a prostaglandin F2-alpha [PGF2a] analogue is used in the treatment of glaucoma. It was found to stimulate skin pigmentation in guinea pigs in addition to its known periocular and iridal pigmentation side effects [2].

Five-fluorouracil [5-FU] is an antimetabolite of the pyrimidine analog type. Fluorouracil is considered a cell cycle-specific for the S-phase of cell division. Activity results from its conversion to an active metabolite in the tissues and includes inhibition of DNA and RNA synthesis [3].

There are two hypotheses that might explain 5fluorouracil induced repigmentation of vitiligo lesions. One is that the drug produces melanocytes colonization in the vitiliginous epidermis by stimulating the division of epidermal melanocytes that migrate into the affected areas after epithelialization of the epidermis occurs. The second proposed that, the melanocytes responsible for repigmentation in vitiligo may be derived from the hair follicles [3].

\section{THE AIM OF THE WORK}

The current study aimed to compare the efficacy of topical latanoprost versus topical 5-fluorouracil after skin dermabrasion in induction of skin repigmentation in stable non-segmental vitiligo.

\section{PATIENTS AND METHOD}

This comparative study was conducted on a total thirty patients with stable vitiligo, 12 males and 18 females. Their age ranged between 18 and 60 years. They were recruited from the outpatient clinic of AlAzhar University Hospitals.

An informed written consent was obtained from all patients.

The inclusion criteria were stable non-segmental vitiligo, both sexes, age above 18 years old. However, patient below 18 years old, with History of koebner phenomenon, pregnant or lactating females, patients received any local or systemic treatment for vitiligo for at least three months before the study, were excluded from the study.

Patients were classified randomly into two groups. Group A, treated with dermabrasion followed by topical application of 5\% 5-fluorouracil under occlusion, and Group B, treated with dermabrasion followed by topical application of latanoprost under occlusion.

Each patient lesions were photograph by a mobile camera before starting the treatment and during the follow-up period. The procedure was performed after topical application of pridocaine [lidocaine $2.5 \%+$ prilocaine $2.5 \%$ ] for 30 minutes. The affected area was cleaned and sterilized with betadine surgical solution followed by alcohol $70 \%$.

The vitiliginous area was treated by dermabrasion with an average speed of $15000 \mathrm{rpm}$ in a uniform horizontal and vertical directions until pinpoint bleeding was observed.

After dermabrasion, 5 -fluorouracil cream $5 \%$ was applied once daily under occlusive dressing for two weeks on one patch and latanoprost drop on another patch once daily under occlusive dressing for two weeks.

Systemic antibiotics to guard against secondary bacterial infection were prescribed. In the next weeks, patients are encouraged to expose themselves moderately to the sun in a slowly increasing dose.

The patients were followed up every two weeks for three months, and photographs before and after therapy were compared. The evaluation of clinical response include the start of repigmentation and degree of pigmentation. The repigmentation responses were expressed qualitatively in accordance with Mina et al. [4] as follow: Poor improvement $=[0 \%-25 \%]$, good improvement $=[26 \%-50 \%]$, very good improvement $=$ $[51 \%-75 \%]$, excellent improvement $=[76 \%-100 \%]$

\section{Statistical analysis:}

Data were collected, revised, coded and entered to 
the Statistical Package for Social Science version 23 $[I B M \cap$ SPSS $\AA$, USA]. The quantitative data were presented as mean, standard deviations and ranges.

The qualitative variables were presented as number and percentages. The comparison between groups with qualitative data were done by Chi-square test and Fisher exact test instead of the Chi-square only when the expected count in any cell found less than 5 . The confidence interval was set to $95 \%$ and the margin of error accepted was set to $5 \%$. Pearson's correlation coefficient was calculated

\section{RESULTS}

This study conducted on 30 patients with nonsegmental stable vitiligo who treated with different modalities. Baseline characteristics of the studied groups presented as: Twelve patients [40\%] were males and eighteen [60\%] were females. Their ages ranged from 18-60 years [mean \pm SD: $37.93 \pm 12.97$ years].

Ten out of thirty patients [33.3\%] had positive family history of vitiligo and 20 patients [66.7\%] had negative family history of vitiligo. Leukotrichia was found in 7 cases [23.3\%] and 23 cases were not associated with leukotrichia [76.7\%].

The Fitzpatrick skin types distributed as the following: one patient has skin Type II [3.3\%], 14 patients with type III [46.7\%] and 15 patients with skin type IV [50.0\%].

Sixty vitiligo patches were studied in this study distributed over the face [n=4], trunk [n=28], extremities [ $n=36]$ and acrofacial [ $n=6]$.

At the end of the $3^{\text {rd }}$ month, patient groups revealed the following results: There was no statistically significant differences between both groups regarding total response to treatment and degree of repigmentation, although there was higher response to treatment in group $\mathrm{A}$ who treated with dermabrasion plus 5\% 5- fluorouracil cream than group $B$ who treated with dermabrasion plus topical latanoprost drops [Table 1].

There was no statistically significant difference as regard satisfaction of patients in both groups, although in both groups there was statistically significant positive correlation between degree of repigmentation and satisfaction of patients [Table 2].

Complications in both groups were the same percentage that lead us to the result that also higher rate of response in group $A$ who treated with dermabrasion plus 5 - fluorouracil than group $B$ who treated with dermabrasion plus topical latanoprost, it did not carry any risk nor increase in susceptibility to complications. In the current work, there were statistically significant negative correlation between duration of the disease and degree of response to treatment [ $p$ value $=0.028]$.

Figures (1 and 2) represented two patients, one of each group.

Figure (1) represented a female patient, 18 years old with a non-segmental stable vitiligo patch, located on her forehead between the eye brows (figure 1a). She was treated by dermabrasion followed by latanoprost, and the last phot showed marked improvement (figure 1b).

Figure 2 describes an abdominal lesion of the same patient, treated by dermabrasion followed by 5 fluorouracil.

Table [1]: Comparison between repigmentation in both groups

\begin{tabular}{|c|c|c|c|c|c|c|}
\hline \multirow[t]{2}{*}{ Repigmentation } & \multicolumn{2}{|c|}{$A(n=30)$} & \multicolumn{2}{|c|}{$B(n=30)$} & \multirow[t]{2}{*}{ Test value* $^{*}$} & \multirow[t]{2}{*}{ P-value } \\
\hline & No. & $\%$ & No. & $\%$ & & \\
\hline Poor & 10 & $33.3 \%$ & 13 & $43.3 \%$ & 0.635 & 0.426 \\
\hline Good & 3 & $10.0 \%$ & 6 & $20.0 \%$ & 1.176 & 0.278 \\
\hline Very Good & 3 & $10.0 \%$ & 4 & $13.3 \%$ & 0.162 & 0.687 \\
\hline Excellent & 14 & $46.7 \%$ & 7 & $23.3 \%$ & 3.590 & 0.058 \\
\hline
\end{tabular}

\footnotetext{
*:Chi-square test
} 


\section{Table [2]: Comparison between satisfactions in both groups}

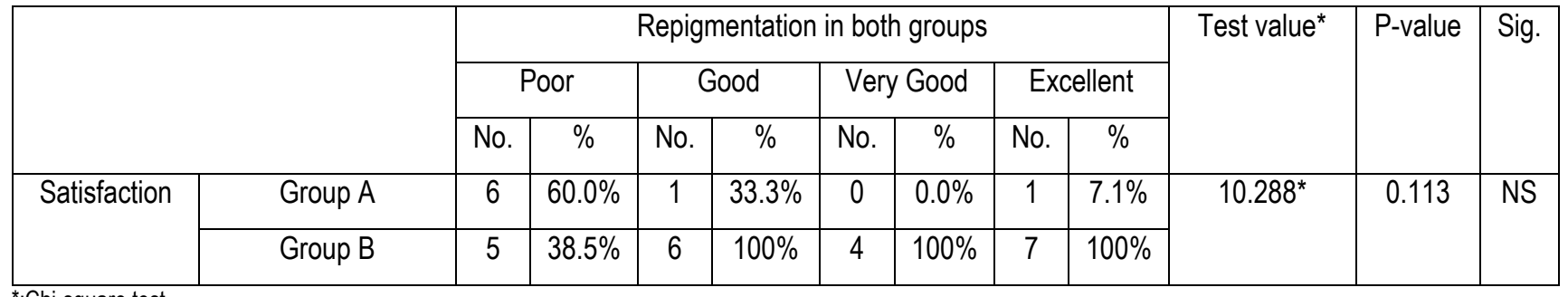
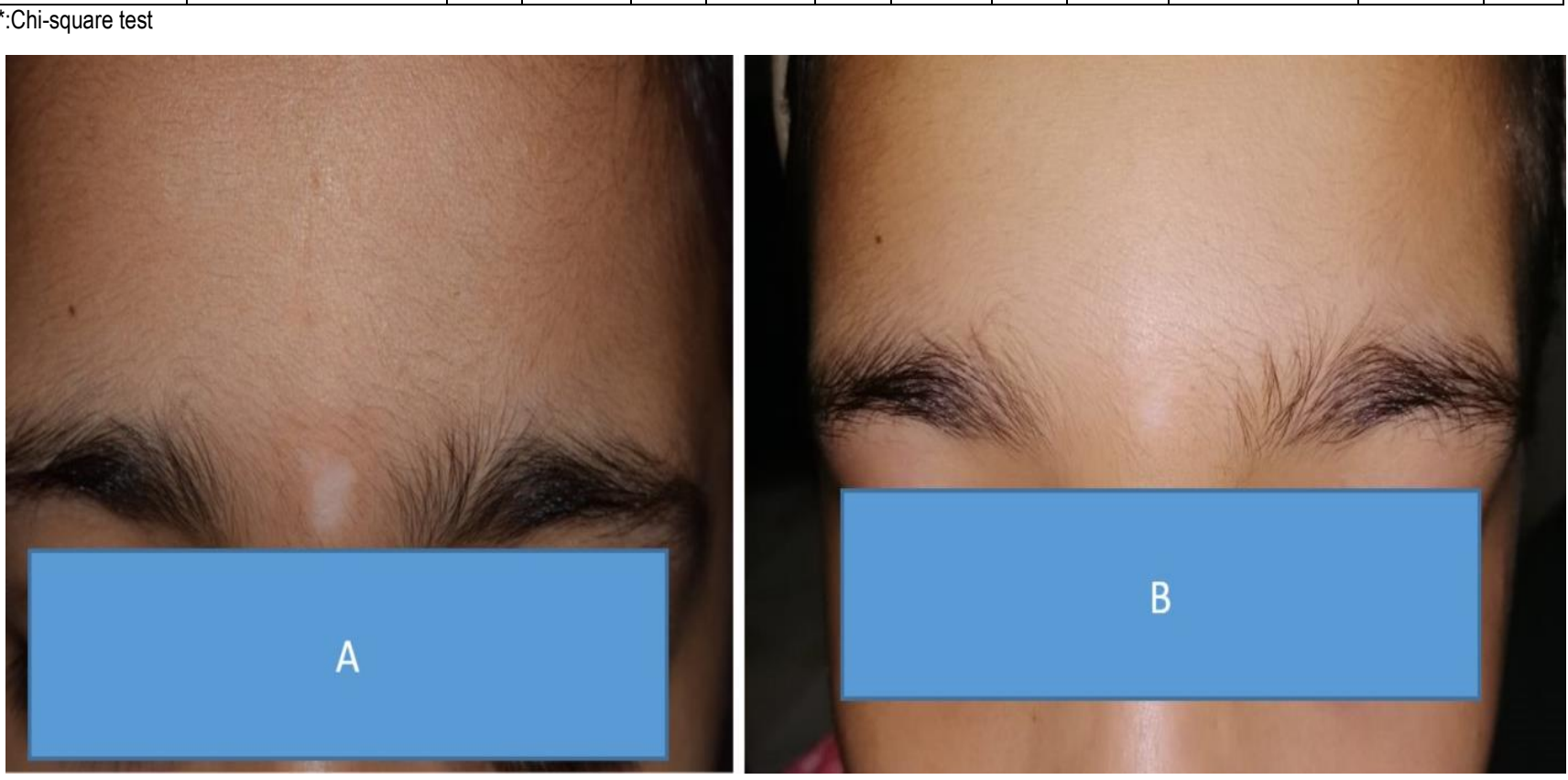

Figure (1): A- The lesion before treatment, and B- showed marked improvement at the last follow up visit, after treatment by latanoprost.

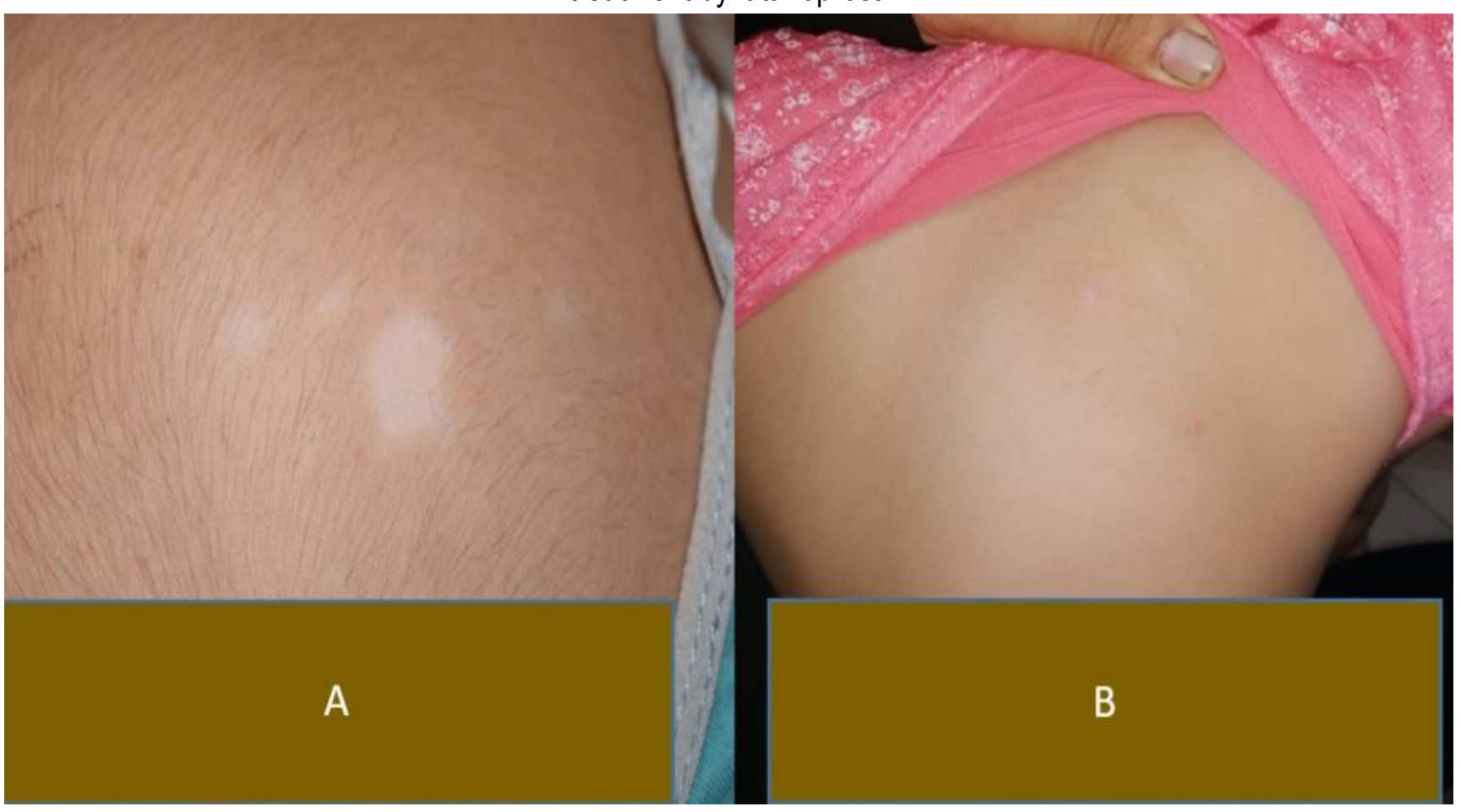

Figure (2): A, pre-treatment and B- after 3 months of treatment by 5 -fluorouracil 


\section{DISCUSSION}

Vitiligo is an acquired pigmentary disorder of unknown etiology. It is characterized clinically by the development of white macules related to chronic and progressive loss of melanocytes from the epidermis and follicular reservoir ${ }^{[4]}$.

Topical $5 \%$ fluorouracil is one of the less popular weapons in the armamentarium for the treatment of vitiligo. It has been tried earlier in combination with dermabrasion and lasers. These procedures, however, often require a special setup with a deep learning curve, and the cost tends to deprive the patients from lower socio economic strata, of these modalities [5].

Although the idea of combination therapy is not a new approach for treatment of vitiligo to our knowledge, this is the first study to date that use dermabrasion combined by topical 5 - fluorouracil in concentration $5 \%$ as a method of improving response rate and hasten the duration of therapy in vitiligo cases resistant to other treatment modalities. This study was conducted on 30 patients with non-segmental stable vitiligo, two vitiliginous patches were treated only in each patient one of them was treated with dermabrasion and topical 5 - fluorouracil $5 \%$ and the other patch was treated with dermabrasion and topical latanoprost.

The insignificant difference between two modalities of treatment is in agreement with Stanimirovic et al. [6] who investigated repigmentation of patients with resistant bilateral symmetrical vitiligo by comparing treatment with narrow band ultraviolet-B and topical $0.005 \%$ latanoprost solution with dermaroller and the other group without dermaroller. They reported no significant difference between both groups and concluded that addition of microneedling seems not to improve the treatment outcome and possibly needs modification.

In the current work, there were statistically significant negative correlation between duration of the disease and degree of response to treatment [ $p$ value $=0.028$.

These results agree with Silpa-Archa et al. [7] who found higher grades of response were more prevalent in patients with recent vitiligo. It may be due to the impending total exhaustion of melanocytes or the total absence of melanocyte reservoirs in the old affected lesions.

The present study results are close to another study reported by Shashikiran et al. ${ }^{8]}$ who reported that the efficacy of topical $5 \% 5$-fluorouracil needling in vitiligo showed excellent repigmentation in $49 \%$ of the patches and very good repigmentation was seen in $26 \%$ of the patches.

Santosh et al. [9] found that microneedling followed by application of 5 -fluorouracil $5 \%$ on vitiligo patches in resistant vitiligo patients, after 3 months showed that about $60 \%$ of patients had hyperpigmentation in the vitiligo lesions with almost complete pigmentation in very small patches; larger ones had less pigmentation and $40 \%$ did not have any pigmentation from the previous state. In contrast our studied group $A$, showed good to excellent response in $66.7 \%$ of cases and $33.3 \%$ poor response only 3 cases [10\%] showed no response.

Also, Vedamurthy et al. [10] reported on three patients of resistant vitiligo. They were subjected to needling with topical 5\% 5-fluorouracil application every two weeks for three months, along with their ongoing therapy. All patients showed excellent repigmentation, but the low number of cases and the different protocols of treatment make comparison of results to be unreliable.

Also our results were close to Mina et al. [4], who compared microneedling plus fluorouracil $5 \%$ with microneedling with tacrolimus and found that $52 \%$ of patients showed good to excellent response [repigmentation $>50 \%$ ] with 5-fluorouracil combined with microneedling.

Our results were close to the results of combination of 5 -Fluorouracil $5 \%$ with dermabrasions which had been studied by Sethi et al. [11] who found marked repigmentation [repigmentation $>50 \%$ ] in $56.7 \%$ of their patients at 4 months.

The response to $5 \% 5$-fluorouracil in this study was better than its combination with Er: YAG laser and NBUVB which reported by Anbar et al. [2] who reported $43.8 \%$ marked response with 5-fluorouracil. However, they used an expensive laser technique that was 
applied on relatively smaller areas of vitiligo [i.e dermabrasion give better response and low cost].

Although 5\% 5-fluorouracil was applied topically after dermabrasion in this study, its efficacy was close to the results of its intradermal injection in 5\% concentration combined with NBUVB which had been studied by Abd El-Samad et al., who reported that $48.3 \%$ of the treated patches showed marked response [repigmentation $>50 \%$ ], this means that dermabrasion can be used as alternative procedure to introduce the drug into the dermis instead of direct intradermal injection [12].

\section{Conclusion:}

The degree of improvement was higher with 5fluorouracil $5 \%$ than latanoprost. However, the difference was statistically non-significant. Patients were satisfied with the degree of improvement in both groups.

\section{Strengths and Limitations}

The strength of this study is its comparative nature which evaluates the effects of treatment in real world settings.

A positive lead to test can inform practice because it provides evidence that treatment or intervention is effective in usual practice.

The weakness of this study was the small sample size and inclusion of single type of vitiligo. Future studies on large-scale group with different kinds of vitiligo are recommended.

\section{Financial and Non-financial Relationships and Activities of Interest}

None

\section{REFERENCES}

1. El-Gayyar MA, Helmy ME, Amer ER, Elsaied MA, Gaballah MA. Antimelanocyte Antibodies: A Possible Role in Patients with Vitiligo. Indian J Dermatol. 2020 Jan-Feb; 65(1):33-37. [DOI: 10.4103/ijd.IJD_344_18].

2. Anbar TS, El-Ammawi TS, Abdel-Rahman AT and Hanna MR . The effect of latanoprost on vitiligo: a preliminary comparative study. Int J Dermatol; 2015,54[5]:587-593.
[DOI: 10.1111/ijd. 12631].

3. El Hawary M, Bassiouny DA, Esmat S, Sobhi R, Saleh MA, AbdelHalim D, et al. Recipient site preparation by cryoblebbing in melanocyte keratinocyte transplantation procedure over the fingers in vitiligo: A pilot study. Dermatol Ther. 2020 Nov;33(6):e14199. [DOI: 10.1111/dth.14199].

4. Mina M, Elgarhy L, Al-Saeid H, Ibrahim Z. Comparison between the efficacy of microneedling combined with 5fluorouracil vs microneedling with tacrolimus in the treatment of vitiligo. J Cosmet Dermatol. 2018 Oct; 17 (5): 744-751. [DOI: 10.1111/jocd.12440].

5. Wu X, Yang Y, Xiang L, Zhang C. The fate of melanocyte: Mechanisms of cell death in vitiligo. Pigment Cell Melanoma Res. 2021 Mar;34(2):256-267. [DOI: 10.1111/pcmr.12955].

6. Stanimirovic A, Kovacevic M, Korobko I, Šitum M, Lotti T. Combined therapy for resistant vitiligo lesions: NB-UVB, microneedling, and topical latanoprost, showed no enhanced efficacy compared to topical latanoprost and NBUVB. Dermatol Ther. 2016 Sep;29(5):312-316. [DOI: 10. 1111/dth.12363].

7. Silpa-Archa N, Weerasubpong $P$, Junsuwan N, Yothachai $\mathrm{P}$, Supapueng $\mathrm{O}$, Wongpraparut $\mathrm{C}$. Treatment outcome and persistence of repigmentation from narrow-band ultraviolet B phototherapy in vitiligo. J Dermatolog Treat. 2019 Nov; 30 (7):691-696. [DOI: 10.1080/09546634.2018.1544409].

8. Shashikiran AR, Gandhi S, Murugesh SB, Kusagur M; Sugareddy. Efficacy of topical $5 \%$ fluorouracil needling in vitiligo. Indian J Dermatol Venereol Leprol. 2018; 84(2):203205. [DOI: 10.4103/ijdvl.IJDVL_386_16].

9. Santosh S K, Sushantika, Mohan L, Gupta A K, Mohammad A, Kumar N. Treatment of Vitiligo with 5-Fluorouracil after Microneedling of the Lesion. Int J Sci Stud. 2018; 5 [11]:125127. [DOI: $10.17354 /$ ijss/2018/56].

10. Vedamurthy M, Moorthy A, Samuel S. Successful treatment of vitiligo by needling with topical 5 fluorouracil. Pigment Disord. 2016; 3:242. [DOI: 10.4172/2376-0427.1000242].

11. Sethi S, Mahajan BB, Gupta RR, Ohri A. Comparative evaluation of the therapeutic efficacy of dermabrasion, dermabrasion combined with topical 5\% 5-fluorouracil cream, and dermabrasion combined with topical placentrex gel in localized stable vitiligo. International Journal of Dermatology; 2007; 46 [8]: 875-879. [DOI: 10.1111/j.13654632.2007.03226.x].

12. Abd El-Samad Z, Shaaban D. Treatment of localized nonsegmental vitiligo with intradermal 5-fluorouracil injection combined with narrow-band ultraviolet. Br J Dermatol Treat. 2012; 23:443-448. [DOI: 10.3109/09546634.2011.579084]. 

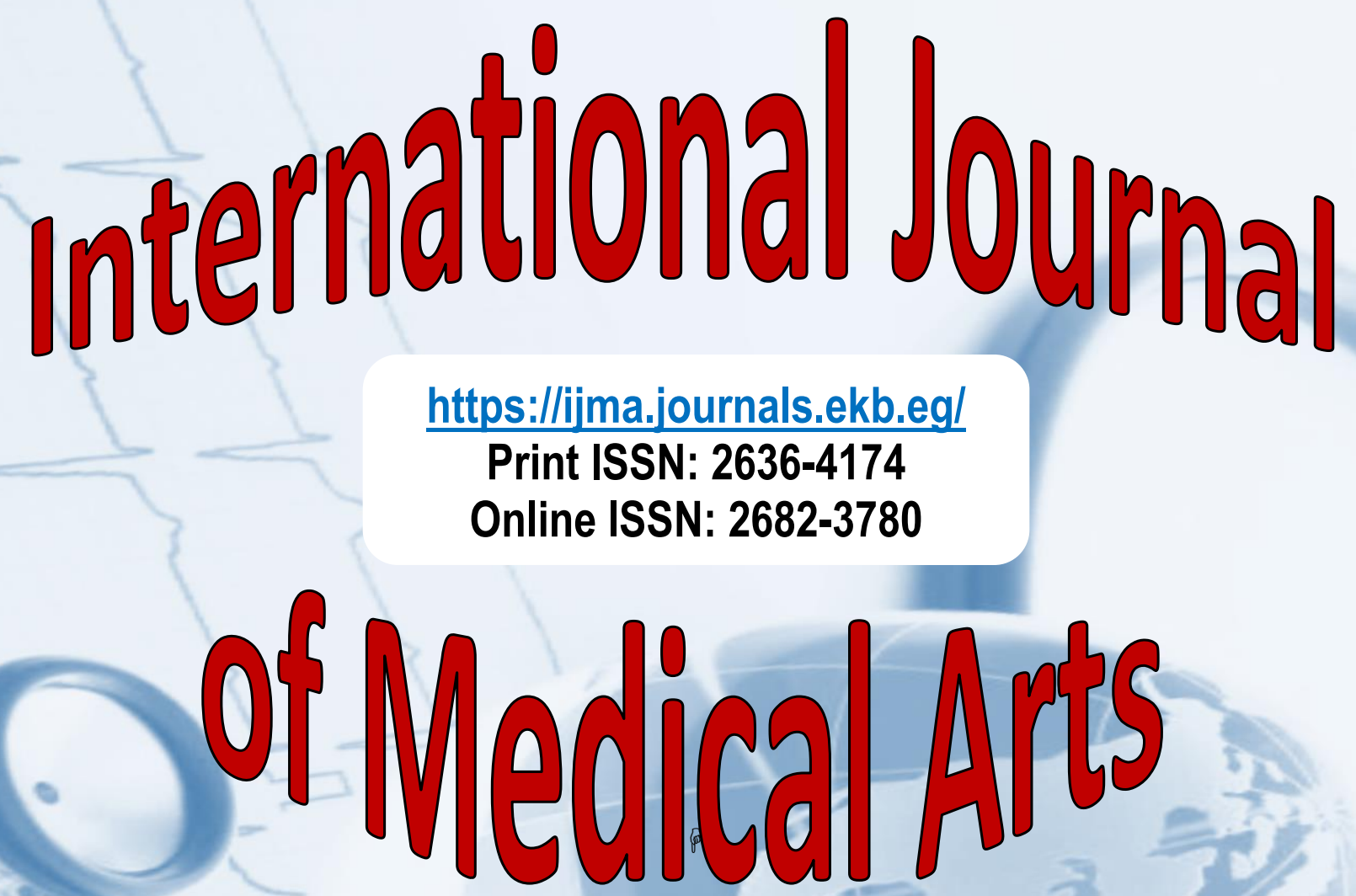